\title{
WHY ARE PLANETARY NEBULAE POOR DISTANCE INDICATORS?
}

\author{
G.A. TAMMANN \\ Astronomisches Institut der Universität Basel, Venusstraße 7, CH-4102 Binningen
}

\begin{abstract}
It is shown from multiple evidence that the $\lambda 5007 \AA$ luminosity of the brightest shells of planetary nebulae has intrinsic scatter, which is well approximated for the brightest objects by an exponential luminosity function, as proposed by Bottinelli et al. (1991). The resulting open-ended luminosity function weakens the usefulness of planetary nebulae as distance indicators.
\end{abstract}

\section{Introduction}

The decisive requirement for any blind application of a distance indicator is that its luminosity (or size) can be determined without any intrinsic scatter. In the presence of intrinsic scatter the actual luminosity (or size) becomes a function of sample size. The problem is particularly severe, if the brightest (or largest) objects of a class are used as distance indicators. Clearly, the brightest objects (with intrinsic scatter) in a giant galaxy are brighter than in a dwarf galaxy, simply because the extreme wings of the luminosity distribution are more completely populated in the former case. The effect is well known for the largest HII regions (Sandage and Tammann 1974a) and for the brightest stars (Sandage and Tammann 1974b; Sandage and Carlson 1988) of late-type galaxies. Therefore the brightest and largest objects are generally expected to be particularly extreme in giant galaxies. Neglect of this effect will always lead to an underestimate of the distance of large galaxies. Since the latter are rare and therefore typically distant, they necessarily lead to a compressed distance scale. In fact, a compressed distance scale can be taken as an unfailing indicator of internal scatter of the distance indicator under consideration.

It has been proposed to use those PNe of a galaxy, which are brightest in the [0 III] $\lambda 5007 \AA$ line, as distance indicators (Jacoby 1989). The situation is here somewhat more favorable, because instead of using the one brightest PN the method relies on the luminosity function (LF) of the brightest tens of PNe. This luminosity function is claimed to drop to zero at a critical luminosity $\mathbf{M}^{*}{ }_{5007}=-4.48$ (Ciardullo et al. 1989). But still the question arises whether a universal luminosity function, which is defined by only a handful of brightest objects, can hold regardless of sample size.

The very steep decrease of PN lifetimes with increasing mass makes it reasonable to postulate an upper cutoff luminosity of the nuclei of PNe. However, the $\lambda 5007 \AA$ luminosity measures the shell, not the nucleus. And there cannot be a one-to-one relation between the nuclear and the nebular luminosities. The relation must be modulated by non-sphericity and other geometrical effects of the shells and, above all, by the very strong evolution of the shell luminosity - particularly of the most massive PNe - of any given PN (cf. Jacoby 1989). Therefore, even if the luminosity of the central star is sharply bounded towards high values, the luminosity of the brightest shells is expected to vary with time and from galaxy to galaxy, and hence to depend also on the sample size, i.e. galaxy size. 


\title{
2. A Comparison of Distances
}

Distances derived by Jacoby and collaborators on the assumption of a constant shape of the $\lambda$ $5007 \AA \mathrm{LF}$ and a unique value of the cutoff magnitude $\mathrm{M}^{*}$, are compiled in Table 1 . They are confronted with modern independent distance determinations. Within $4 \mathrm{Mpc}$ the PNe can reproduce the high-accuracy distances from Cepheids to within $\sim 10 \%$. But beyond the PN distance scale is progressively compressed. At the Virgo cluster the distance discrepancy amounts to a factor of 1.4 .

TABLE 1. Distances from PNe and Other Methods

\begin{tabular}{llllll}
\hline & $\begin{array}{l}(\mathrm{m}-\mathrm{M})^{\circ} \\
\text { from PNe } \\
(2)\end{array}$ & $\begin{array}{l}\text { Source } \\
(1)\end{array}$ & $\begin{array}{l}(\mathrm{m}-\mathrm{M})^{\circ} \\
\text { from others } \\
(4)\end{array}$ & $\begin{array}{l}\text { Method } \\
(5)\end{array}$ & $\begin{array}{l}\Delta(\mathrm{m}-\mathrm{M}) \\
(6)\end{array}$ \\
\hline LMC & 18.44 & 1 & 18.50 & Cepheids (7) & -0.06 \\
SMC & 19.09 & 1 & 18.87 & Cepheids (7) & +0.22 \\
M31 & 24.26 & 2 & 24.44 & Cepheids (7) & -0.18 \\
M81 & 27.72 & 3 & 27.59 & Cepheids (7) & +0.13 \\
Leo & 30.02 & 4 & 30.50 & Source 8 & -0.48 \\
NGC 1023 & 29.97 & 5 & $30.96:$ & Source 9 & $-0.99:$ \\
NGC 891 & & & & Source 10 & -0.74 \\
Virgo & 30.90 & 6 & 31.64 & &
\end{tabular}

\begin{abstract}
Sources. 1. Jacoby et al. (1990). 2. Ciardullo et al. (1989a). 3. Jacoby et al. (1989). 4. Ciardullo et al. (1989b). 5. Ciardullo et al. (1991). 6. Jacoby et al. (1990a). 7. Madore and Freedman (1991). 8. The difference between the Leo Group and the Virgo cluster is determined from metallicity-corrected surface brightness fluctuations (Tammann 1992), PNe corrected for sample size effects (Bottinelli et al. 1991), globular clusters (Harris 1990), and the $D_{n}-\sigma$ relation (Faber et al. 1989). 9. The distance is determined from the recession velocity $\mathrm{v}_{220}=855 \mathrm{~km} \mathrm{~s}^{-1}$ (corrected for a Virgocentric infall model) of the group and $\mathrm{H}_{\mathrm{o}}=55 \mathrm{~km} \mathrm{~s}^{-1}$ $\mathrm{Mpc}^{-1}$. Due to peculiar motions the modulus may be off by $\sim 0 .^{\mathrm{m}} 4$. 10 . From globular clusters, novae, supernovae, the $D_{n}-\sigma$ relation, $21 \mathrm{~cm}$ line widths, and the scale length of the Galaxy and M31 (Tammann 1992, and references therein).
\end{abstract}

The significance of the PN distance scale being too short has recently been enhanced by new determinations of the Virgo distance. Expanding-photosphere models of two supernovae of type II provide a Virgo modulus of $(\mathrm{m}-\mathrm{M})=31.71 \pm 0.26$ (Schmidt et al. 1992). A direct luminosity calibration of the type I supernova 1937C through the Cepheids in the parent galaxy IC 4182 (Sandage et al. 1993), if applied to the 10 supernovae of that type in the Virgo cluster, provides a Virgo modulus of $(\mathrm{m}-\mathrm{M})=31.64 \pm 0.22$. These new determinations are again much higher than the distance from $\mathrm{PNe}$.

In a Critical Review (Jacoby et al. 1992), which defies its name, much emphasis is given to the fact that the Virgo distance from PNe agrees with that from the surface brightness fluctuation method (Tonry 1991). Unfortunately the latter method is dominated by metallicity effects and does not yield useful distances (Tammann 1992).

The expectation that the compression of the PN distance scale is due to an increase of the $\lambda 5007$ $\AA$ luminosity of the brightest PNe with the sample (galaxy) size has first been substantiated by Bottinelli et al. (1991), who have shown that the PN distance modulus $\mu$ of individual Virgo galaxies depends on the apparent (and hence absolute) magnitude of the galaxy (Fig. 1).

To elaborate the dependence of the brightest PNe on the galaxy size, the data in Table 2 were compiled. Column 2 lists the absolute bolometric magnitude of the galaxy, or in cases where only a fraction of the galaxy was surveyed, the corresponding magnitude of that fraction. The data were taken from the sources 1-6 in Table 1 and adjusted to the distances adopted in col. 4 of Table 1. 


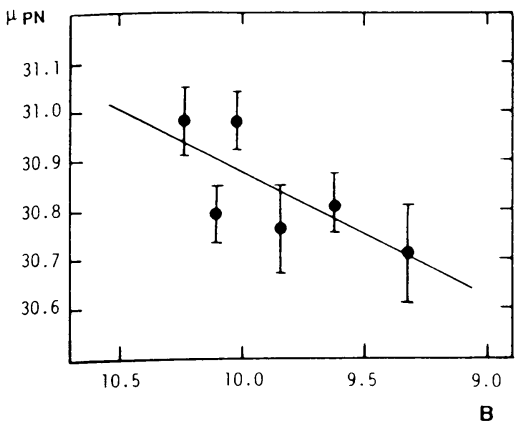

Figure 1. PN distance moduli $\mu$ of individual Virgo galaxies against their apparent (B) magnitude. (From Bottinelli et al. 1991).

TABLE 2. Bolometric Magnitudes of Surveyed Galaxy Populations, Absolute Magnitudes of the Brightest PNe, and Nominal Deathrates of Stars

\begin{tabular}{llll}
\hline$(1)$ & $\begin{array}{l}\mathrm{M}^{\mathrm{o}}{ }_{\text {bol }} \\
(2)\end{array}$ & $\begin{array}{l}\mathrm{M}^{\mathrm{o}}{ }_{5007}(3) \\
(3)\end{array}$ & $\begin{array}{l}\log \alpha_{25}+9 \\
(4)\end{array}$ \\
\hline LMC & - & -4.33 & - \\
SMC & - & -3.79 & - \\
M31 & -19.74 & -4.41 & 1.19 \\
NGC 185 & -15.93 & -3.49 & 1.38 \\
NGC 205 & -16.18 & -3.67 & 1.70 \\
M32 & -16.81 & -3.77 & 1.32 \\
M81 & $(-20.95)$ & -4.54 & $(1.26)$ \\
NGC 3377 & -19.36 & -4.98 & 1.38 \\
NGC 3379 & -20.67 & -5.13 & 1.13 \\
NGC 3384 & -20.04 & -4.91 & 1.39 \\
NGC 1023 & $(-19.98)$ & -5.50 & 0.94 \\
NGC 891 & - & -5.34 & - \\
NGC 4374 & -21.25 & -5.44 & 0.92 \\
NGC 4382 & -21.58 & -5.61 & 1.00 \\
NGC 4406 & -22.28 & -5.75 & 0.83 \\
NGC 4472 & -22.19 & -5.24 & 0.52 \\
NGC 4486 & -21.95 & -5.74 & 0.62 \\
NGC 4649 & -22.08 & -5.11 & 0.51 \\
& & & \\
\hline
\end{tabular}

The mean absolute $\lambda 5007 \AA$ magnitude of the three brightest PNe in column 3 includes all objects found by Jacoby and collaborators. These authors had excluded some of the brightest objects for the sole reason that they do not fit under their adopted LF. But because this LF is in question, all objects, which are bright in the $\lambda 5007 \AA$ line, must be considered as shells of PNe, unless proven to the contrary.

The data of Table 2 are plotted in Fig. 2. They expose a pronounced correlation of the mean absolute magnitude $\mathrm{M}^{\mathrm{o}}{ }_{5007}(3)$ of the three brightest planetry shells with the bolometric magnitude of the surveyed population. The correlation is illustrated in the Figure by a line with slope 0.25 . This slope necessarily follows if the bright end of the PN LF has the form $\mathrm{N}(\mathrm{M}) \propto 10^{1.6 \mathrm{M}}$. This form of the LF approximates indeed the available data well, as shown in the next Section. 


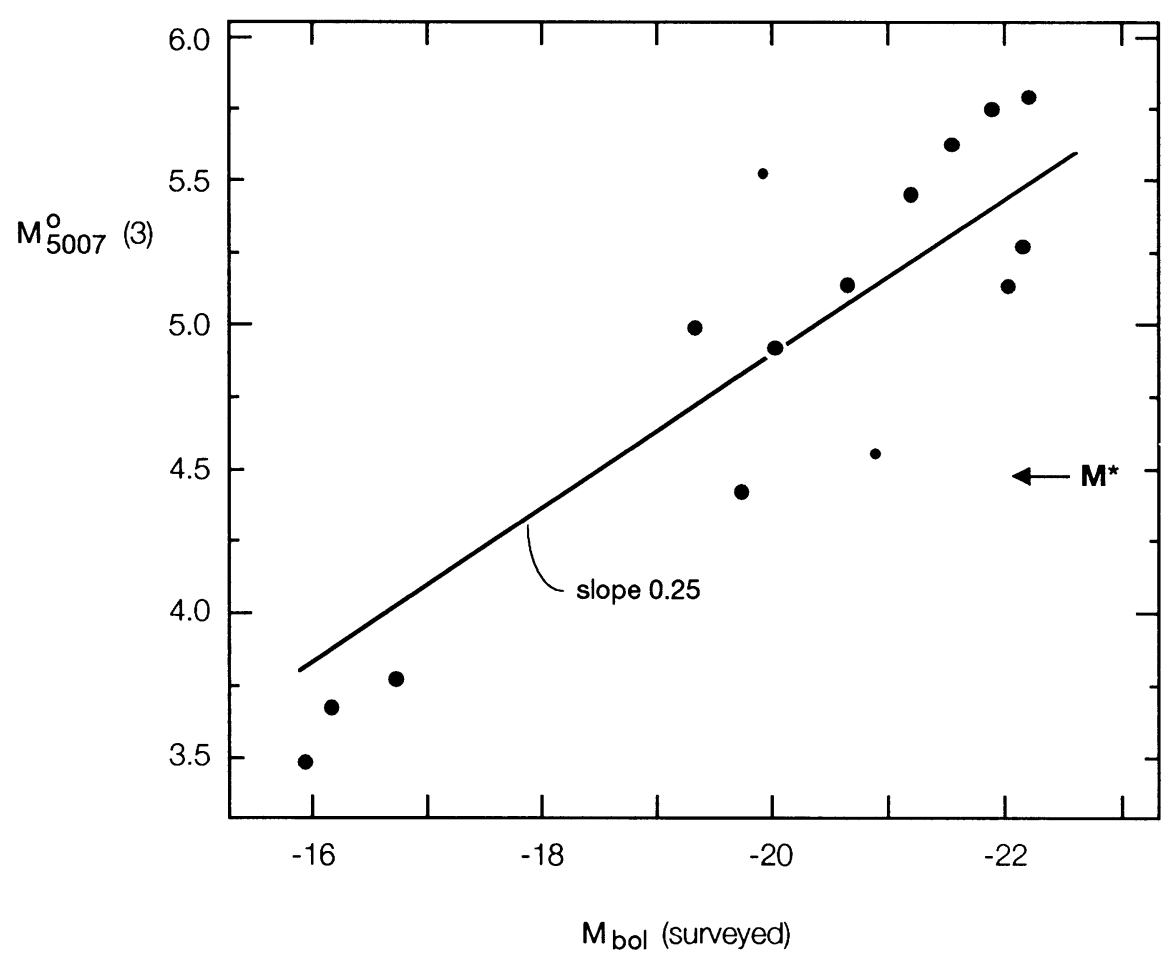

Figure 2. The dependence of the mean absolute magnitude of the three brightest PN shells on the sample size, i.e. $\mathrm{M}^{\circ}$ bol (surveyed). The slope of 0.25 of the drawn line follows from $\log \mathrm{N}(\mathrm{M}) \propto$ 1.6 M. Small symbols have uncertain $\mathrm{M}^{\circ}{ }_{\text {bol. }}$.

\section{The Luminosity Function of Bright PNe}

The largest available body of data to determine the bright end of the (differential) $\lambda 5007 \AA$ LF of planetary shells is provided by the six Virgo ellipticals which were searched for PNe, and which are assumed to lie at the same distance. The combined data are shown in Figure 3.

Two fits are shown to the data in Fig. 3. The one is the luminosity function as proposed by Jacoby and collaborators with a cutoff magnitude $\mathrm{M}^{*}$, the other one is an exponential function of the form $\mathrm{N}(\mathrm{M}) \propto 10^{1.6 \mathrm{M}}$ as proposed by Bottinelli et al. (1991). Clearly the latter is more realistic by giving a finite probability to the observed objects brighter than $\mathrm{M}^{*}$. The finite, albeit small probability of overluminous shells reaches order of unity in large samples. In fact the LF of Bottinelli et al. (1991) very well explains the observed correlation between the brightest PN shells and the sample size (cf. Fig. 2).

The data are too sparse to allow an exact determination of the shape of the LF. The exponential function of Bottinelli et al. (1991) is a useful working model at least for the extreme end of the luminosity function, because it allows for the obvious intrinsic luminosity scatter of the brightest planetary shells. If the exponential LF is adopted here, this is not to say, that it holds over a wider luminosity interval nor that it reflects the final shape of the LF. 


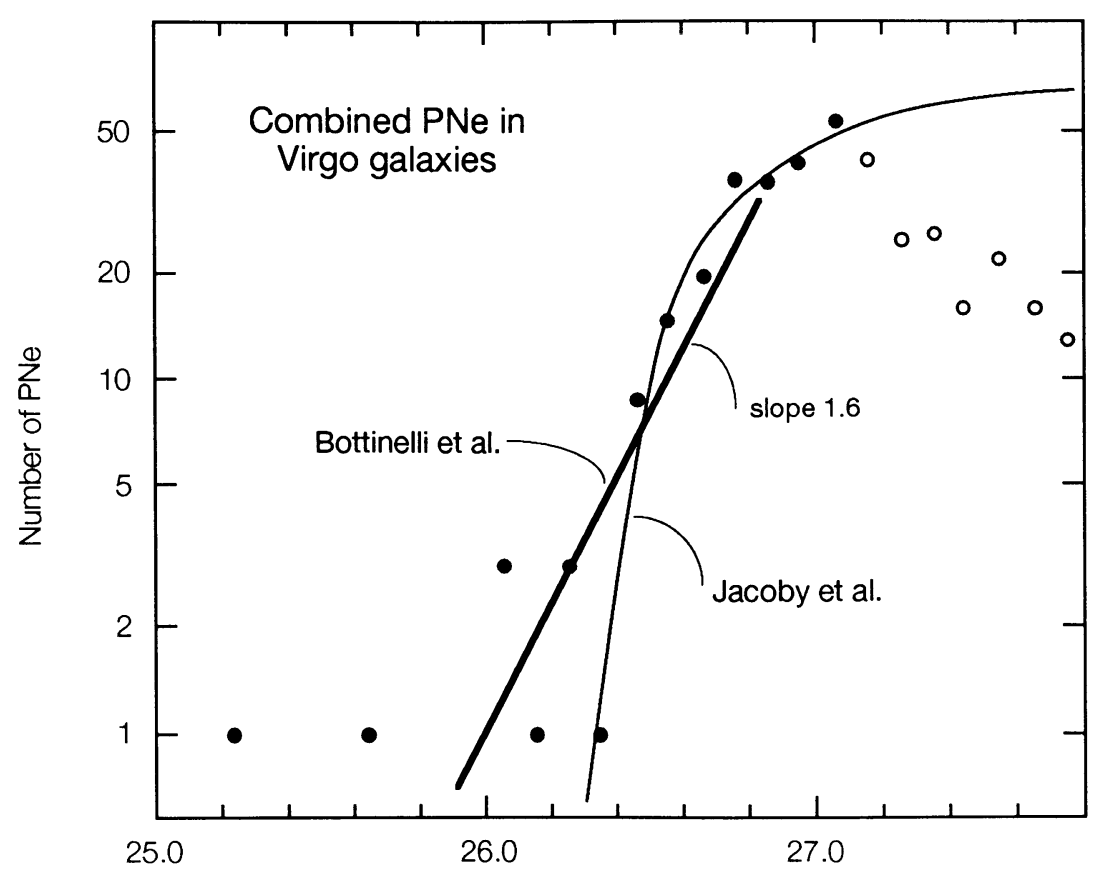

Apparent $\lambda 5007$ magnitude

Figure 3 . The observed differential $\lambda 5007 \AA$ luminosity function of planetary shells for a combined sample of six elliptical galaxies in the Virgo cluster. Open symbols are affected by incompleteness.

The great impact of the shape of the extreme end of the luminosity function on the distance scale comes from the fact, that the surveyed populations are widely different and increase in size with increasing distance. At the distance of the Virgo cluster the surveyed samples are on average seven times larger than that of the calibrator M31.

The expectation that the luminosity of the three brightest objects decreases, when the population size decreases, can be checked in the Virgo galaxies by defining random subsamples, which agree in population size, for instance, with that of the calibrator M31. Monte Carlo calculations show that $\mathrm{M}^{\mathrm{o}}{ }_{5007}(3)$ becomes $0{ }^{\mathrm{m}} 6$ fainter on average for the subsamples of appropriate size than for the total surveyed population of the six Virgo ellipticals. This immediately explains why the use of the artificial cutoff magnitude $\mathbf{M}^{*}$ must lead to a compressed distance scale.

Another puzzle of the compressed distance scale is the large galaxy-to-galaxy variation of the specific PN number $\alpha_{2.5}$, where $\alpha_{2.5}$ is the number of PNe per $10^{9} \mathrm{~L}_{\odot}$ within the brightest 2.5 magnitude interval. The variation of $\alpha_{2.5}$, which is proportional to the specific stellar death rate, is expected to be roughly constant in elliptical galaxies, but Jacoby and collaborators derived values which differ by a factor of 6 . The $\log \alpha_{2.5}$ values derived from the presently adopted distance scale are listed in Table 2, column 4, and are plotted in Fig. 4 against $\mathrm{M}^{\mathrm{o}}{ }_{\text {bol }}$ of the surveyed population. Also here is a clear variation of $\alpha_{2.5}$ with $\mathrm{M}^{\mathrm{o}}{ }_{\text {bol }}$, but the trend is well represented by a line of slope 0.3 , which is exactly expected if $\mathrm{N}(\mathrm{M}) \propto 10^{1.6 \mathrm{M}}$ (cf. Bottinelli et al. 1991). Only the dwarf 


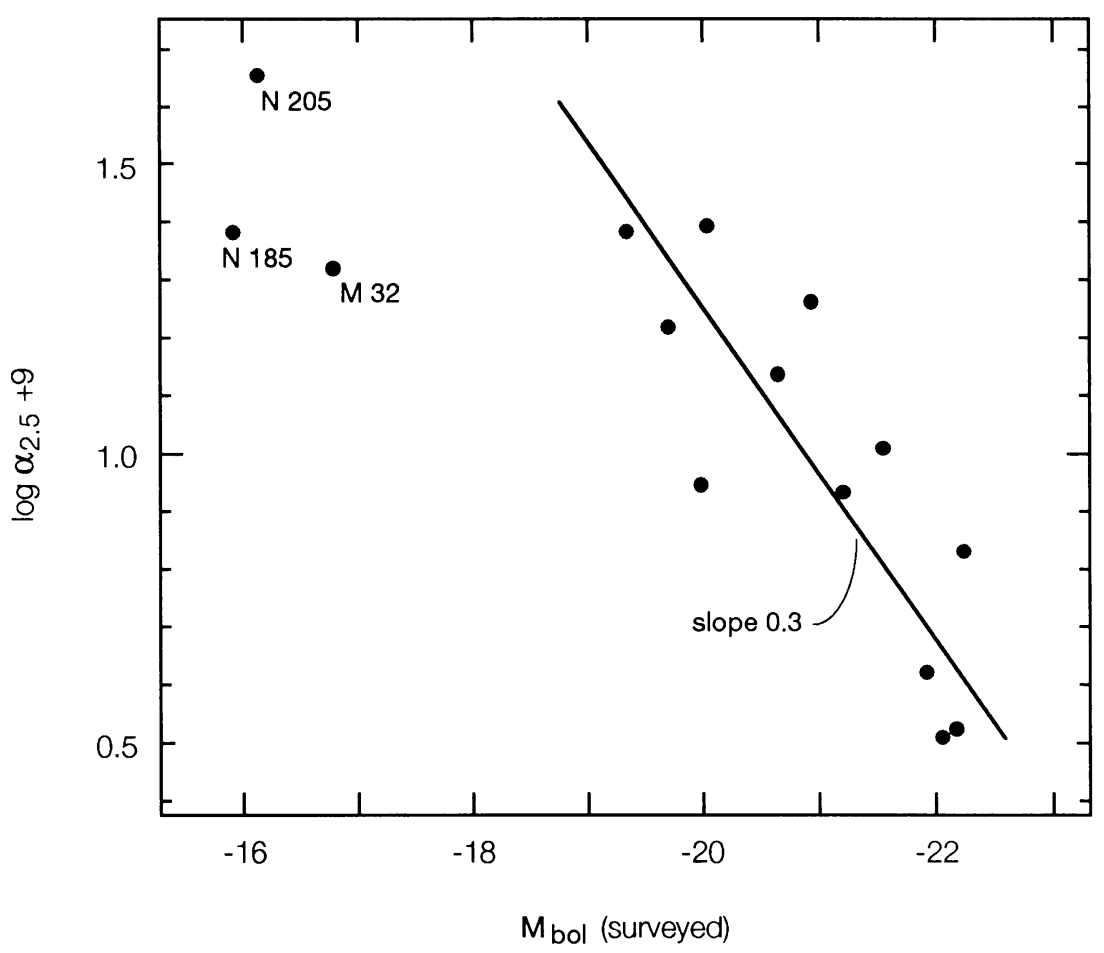

Figure 4. $\log \alpha_{2.5}$ versus $\mathrm{M}^{\mathrm{o}}$ bol of the surveyed population. The slope of 0.3 of the drawn line follows from $\log \mathrm{N}(\mathrm{M}) \propto 1.6 \mathrm{M}$.

ellipticals fall off the line, since their PN numbers are so small that they do not populate the bright exponential tail of the luminosity function. In the case of an exponential LF the stellar death rates as measured by $\alpha_{2.5}$ cannot be compared directly. In large galaxies $\alpha_{2.5}$ measures the small formation rate of overluminous shells, whereas in smaller galaxies it measures the (high) formation rate of more average shells.

Ferguson and Davidsen (1992) have pointed out a correlation of the original $\alpha_{2.5}$ values with the far-UV flux of elliptical galaxies. This correlation is maintained also with the new values of $\alpha_{2.5}$ in Table 2. In either case the correlation is surprising because $\alpha_{2.5}$ has no clear physical meaning. Unfortunately the integration of the total PN numbers down to a uniform limiting luminosity is not possible, because the exponential shape of the luminosity shape holds only for the brightest part.

\section{Conclusions}

The assumption of a cutoff magnitude $\mathrm{M}^{*}$ of the $\lambda 5007 \AA$ LF of PN shells leads to a Virgo cluster distance of $\sim 15 \mathrm{Mpc}$, which is to be compared with all independent evidence requiring $21 \pm 2$ $\mathrm{Mpc}$. The obvious reason for the compressed distance scale is the intrinsic scatter of the $\lambda 5007 \AA$ luminosity of the brightest shells. This makes the distances from PNe sensitive to the population size, and leads to systematic distance underestimates for distant and hence large galaxies.

Even if the masses of planetary nuclei obeyed a delta function, some luminosity scatter of the brightest shells would be expected simply because of their strong luminosity evolution. The prob- 
ability of catching a PN during its brightest shell phase increases, of course, with increasing sample size. This effect is strongly enhanced if the planetary masses have a finite, albeit narrow range. Jacoby and collaborators assumed a Gaussian mass function with $\mathfrak{m}=0.61 \pm 0.02 \mathfrak{m}_{\odot}$. A central star, with three standard deviations above the mean mass, produces a short-lived, extremely bright shell (cf. Jacoby 1989), whose detection probability clearly depends on population size.

The intrinsic scatter of the luminosity of the brightest shells produces a LF which - as Bottinelli et al. (1991) have shown - is well approximated at the extreme tail by $\mathrm{N}(\mathrm{M}) \propto 10^{1.6 \mathrm{M}}$. This form indeed explains the observed distance dependence of Virgo ellipticals on their luminosity (Fig. 1), the luminosity increase of the three brightest shells with $\mathrm{M}^{\circ}{ }_{\text {bol }}$ of the surveyed population (Fig. 2), and the wide variation of the specific PN numbers $\alpha_{2.5}$ with $\mathbf{M}^{\circ}{ }_{\text {bol }}$ (Fig. 4).

Planetary nebulae as distance indicators are still plagued by other problems. The calibration of the (artificial) cutoff magnitude $\mathbf{M}^{*}$ rests on the PNe in the bulge of an Sb galaxy (M31), whereas it is applied to E galaxies. Jacoby et al. (1990b) have argued that $\mathbf{M}^{*}$ is independent of Hubble type, because they could recover the distances of LMC and SMC, and Ciardullo et al. (1991) found the same distance for the neighboring galaxies NGC 1023 (SB0) and NGC 891 (Sb). But this may be the result of the interplay of three varying parameters: population size, metallicity, and evolutionary history. In the case of the Magellanic Clouds and NGC 891 not even $\mathbf{M}^{*}{ }_{\text {bol }}$ of the surveyed population has been specified. Metallicity variations alone affect $\mathbf{M}^{*}$ by $\sim 0{ }^{\mathrm{m}} 3$ according to Ciardullo and Jacoby (1992). Moreover Dopita et al. (1992) have shown from theoretical models that the evolutionary history has a very strong influence on the shell luminosity. A variation of the ages of bulge stars in spirals from 0.8 to $5.0 \mathrm{Gyr}$ affects the $\lambda 5007 \AA$ luminosity by $0 . \mathrm{m} 44$, and the age variation from 5.0 to $10.0 \mathrm{Gyr}$ of the stars in (Virgo) ellipticals causes an additional luminosity change by $0 .{ }^{\mathrm{m}} 59$ !

No stringent observational test of the claimed stability of $\mathbf{M}^{*}$ has so far been attempted. This would involve about ten E galaxies in the Virgo cluster of widely different luminosity. The expectation is that their brightest PN shells follow the same relation as in Fig. 2. If indeed the relation is confirmed, PNe are weakened as distance indicators, because their brightness and the sample size depend on distance (cf. Bottinelli et al.). Moreover, the problem of the local calibration remains, because no fundamental distance is known to any normal $\mathrm{E}$ galaxy.

As a final note it is added that the observation of PN shells through the $\lambda 5007 \AA$ filter is even at the distance of the Leo group by no means a simple matter. Ongoing work on sub-arcsec observations of NGC 3379 by S. Wagner and collaborators recovers - as a preliminary result - only about $80 \%$ of the objects of Ciardullo et al. (1989b), but finds roughly $50 \%$ of additional objects. Independent checks of the existing lists of PNe are in any case desirable.

Acknowledgement: Support of the Swiss National Science Foundation is gratefully acknowledged.

\section{References}

Bottinelli, L., Gouguenheim, L., Paturel, G., and Teerikorpi, P. 1991, A.A. 252, 550.

Ciardullo, R., and Jacoby, G.H. 1992, Ap.J., in press.

Ciardullo, R., Jacoby, G.H., and Ford, H.C. 1989b, Ap.J. 344, 715.

Ciardullo, R., Jacoby, G.H., Ford, H.C., and Neill, J.D. 1989a, Ap.J. 339, 53.

Ciardullo, R., Jacoby, G.H., and Harris, W.E. 1991, Ap.J. 383, 487.

Dopita, M.A., Jacoby, G.H., and Vassiliadis, E. 1992, Ap.J. 389, 27.

Faber, S.M., Wegner, G., Burstein, D., Davies, R.L., Dressler, A., Lynden-Bell, D., and Terlevich, R.J. 1989, Ap.J.Suppl. 69, 763.

Ferguson, H.C., and Davidsen, A.F. 1992, preprint.

Harris, W.E. 1990, P.A.S.P. 102, 966.

Jacoby, G.H. 1969, Ap.J. 339, 39.

Jacoby, G.H. et al. 1992, P.A.S.P. 104, 599.

Jacoby, G.H., Ciardullo, R., and Ford, H.C. 1990a, Ap.J. 356, 332.

Jacoby, G.H., Walker, A.R., and Ciardullo, R. 1990b, Ap.J. 365, 471.

Jacoby, G.H., Ciardullo, R., Ford, H.C., and Booth, J. 1989, Ap.J. 344, 704. 
Jacoby, G.H., Walker, A.R., and Ciardullo, R. 1990, Ap.J. 365, 471.

Madore, B.F., and Freedman, W.L. 1991, P.A.S.P. 103, 933.

Sandage, A., and Carlson, G. 1988, A.J. 96, 1599.

Sandage, A., Saha, A., Tammann, G.A., Panagia, N., and Macchetto, F. 1993, Ap.J.Letters, in press.

Sandage, A., and Tammann, G.A. 1974a, Ap.J. 194, 559.

Sandage, A., and Tammann, G.A. 1974b, Ap.J. 191, 603.

Schmidt, B.P., Kirshner, R.P., and Eastman, R.G. 1992, Ap.J., in press.

Tammann, G.A. 1992, Physica Scripta T 43, 31.

Tonry, J.L. 1991, Ap.J.Letters 373, L1. 